\title{
Embedding and Identifying Medical Information on Round- Shaped Vials Using Circular Dot Pattern Code and Topological Graph Matching-Based Detection Scheme
}

\author{
Jae Youn Shim ${ }^{1}$ and Seong Whan Kim ${ }^{2 *}$ \\ ${ }^{1}$ Depart of Computer Engineering, Kyungdong University, Gangwondo, \\ 24764, Korea \\ ${ }^{2}$ School of Computer Science, University of Seoul, Seoul, 130-743, Korea \\ ${ }^{1}$ simpo@kdunivs.ac.kr, ${ }^{2 *}$ swkim7@uos.ac.kr
}

\begin{abstract}
Administration of medicines (especially guarantee of manufacture date and expiry date) is an important issue for patient safety in medical applications. Medical applications have a strong need for embedded tags on medical bottles to facilitate medicine management. However, $1 D$ barcode cannot fulfill the required information capacity. The $2 D$ barcode can support information capacity; however, it cannot meet the commercial level recognition rate when it is being used on curvature surfaces. On the other hand, the RFID tags are 100 times more expensive than barcode systems and are also difficult to embed on curvature surfaces. In this paper, a robust circular dot pattern code $(C D P C)$ for tagging medical information on cylindrical and round type bottles for identification and management is proposed. Originally, CDPC was designed using the topological layout of dot code, and is well suited to recognizing tags in curvature environments. The different tagging mechanisms were presented in order to illustrate its importance on the administration of medicines. Experimental results show that the CDPC outperformed $2 D$ barcode in its recognition rate on curvature including cylindrical and round shape.
\end{abstract}

Keywords: Medical package, Code recognition system, Circular Dot pattern code

\section{Introduction}

In medical applications, taking an adequate medicine is important for the patient's health. Abuse of the medicine may be harmful to the health of the patient. The pharmaceutical packaging market has been constantly growing, with annual growth of at least $5 \%$ per annum in the past few years. According to Jain and Nayak, the size of the pharmaceutical packaging market is estimated to be over $\$ 20$ billion in 2008 [1]. The pharmaceutical packaging product types include prescription bottles, vials, ampoules, and syringes with cartons. Prescription bottles contain medicine prescribed by physicians. A vial is one type of prescription bottle and is a small glass or plastic bottle. An ampoule is a small, sealed vial that is used to contain and preserve a sample. A syringe is a pump comprising a plunger tightly fitting in a tube. A carton means a box or container which is generally made of paperboard and occasionally made of corrugated fiberboard. Most pharmaceutical packages are supplied packed in cartons. Packaging means a collection of components which surrounds a pharmaceutical product during the time of its production to its use. Medical information of manufacture date and expiry date are important for patient safety. Since 2013, the Korean Government enacted that specialty

* Corresponding Author 
pharmaceuticals should attach 2D barcodes or RFID for denoting manufacture date and expiry date for user safety [2]. However, 2D barcode including QR code is difficult to recognize when it is attached to cylindrical and round type surfaces. RFID can support large capacity of information, however, it is 100 times more expensive than barcode systems, and is also easily vulnerable to simple impact.

In this paper, the CDPC was used to attack the affine or perspective transformation of tagging information when the tag is attached to the round shaped vials. Because CDPC basically uses topology-based graph matching approach instead of pixel-based approach, it can be more robust to any kind of curvature-based tagging.

The remainder of this paper is organized as follows: Section 2 reviews barcode, QR code, RFID, and CDPC related works; Section 3 illustrates the tagging mechanisms; the design of the CDPC and topological graph matching for embedded tags on round-shaped vials is discussed in Section 4; Section 5 shows the experimental results of cylindrical and round type bottle tag using CDPC; and the concluding remarks on Section 6.

\section{Previous Works}

A tag is a non-hierarchical term or keyword assigned to specific information. This sort of metadata helps explain the item, and you can find it again by searching or browsing. The tags are usually selected in informal and personal manner by the item creator or viewer, depending on the system. A tag should be easy to recognize, even if the tag is attached to the curved surface for cylindrical and round type medical bottles as shown in Figure 1 .
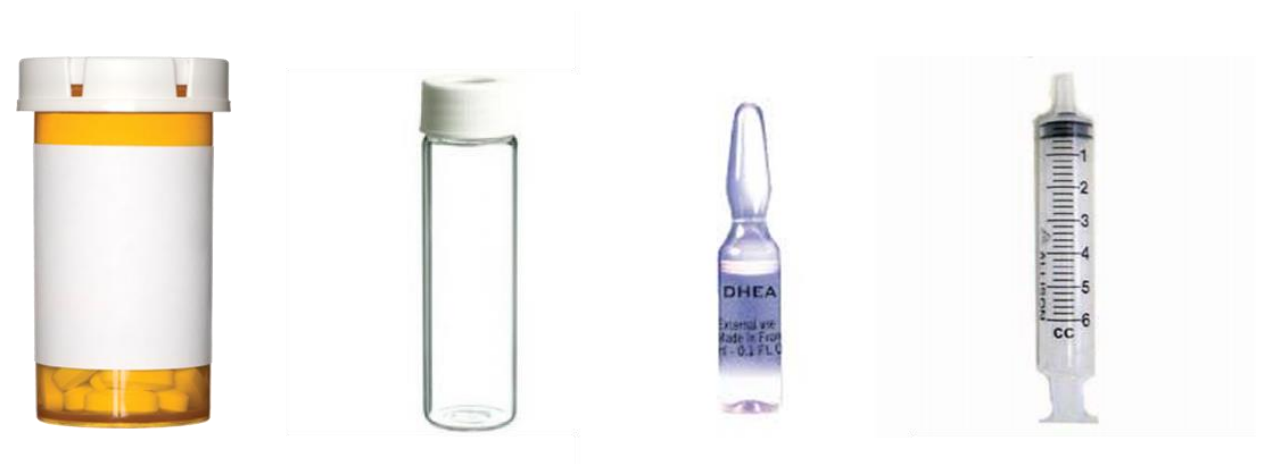

Figure 1. Prescription Bottle, Vial, Ampoule, and Syringe

Figure 1 shows the shape of a prescription bottle, a vial, an ampule, and a syringe. Prescription bottles have various shapes, including cylindrical/round, square, rectangular, oval, etc. A vial is one type of prescription bottle and can be a small glass or plastic bottle, often used to store medication as liquids, powders, or capsules. An ampoule is an enclosed small vial used to store and preserve solid or liquid samples. A syringe is a pump comprising a plunger tightly fitting in a tube. The plunger can be pulled and pushed inside a cylindrical tube. Their shape type is cylindrical and round.

1D barcodes have been developed and used for distribution in fields such as retail markets and the military. 1D barcode is composed of patterns such as guard bars that indicate the initial, final, and central positions of the image code, and data patterns that encode the numeric data and checksum digit [3]. 1D bar code patterns are expressed in terms of relative thickness ratios of parallel black and white bars. The mapping between the message and the barcode is named as a symbology. The specifications of the symbology include the encoding of characters/single digits in the message, the encoding of start and stop markers into spaces and bars, the quiet zone size required before and 
after the barcode, and the calculation of the checksum. It is mandatory that the quiet zone being at least 10 times the width of the narrowest bar/space element should be on the left and right of the barcode. Start/stop character must be the first and last character appearing in the complete barcode. Inter- character gaps are the space between characters. Thus, by sampling the pixels on scan lines placed across the patterns, the decoder can recognize the code if at least one scan line covers all the patterns. After reading all the patterns, the decoder verifies the result by using the checksum digit in the data patterns.

QR codes, two-dimensional barcodes created by Denso-Wave, are the most popular type of two-dimensional codes [4]. They are currently used in broader contexts, and their use is increasing to include convenience-oriented applications for mobile phone users. According to Denso-Wave, QR codes that store addresses and URLs can appear in a variety of advertisements, business cards, or any object that would allow a user to obtain information on a particular item [5]. QR codes are capable of handling various data types including numeric and alphabetic characters, Japanese characters, symbols, and binary and control codes. Forty versions of QR code symbols are available, you can encode up to 7,089 characters in each symbol. Version 1 specifies $21 \times 21$ modules. Each version of the symbol adds 4 modules on each side until reaching version 40, which contains $177 \times 177$ modules. Moreover, QR codes have error correction capability. Even in the event of a symbol partially obscured by dirt or damage, QR codes can correct errors in approximately $7 \%, 15 \%, 25 \%$, and $30 \%$ of code words at levels $\mathrm{L}, \mathrm{M}, \mathrm{Q}$, and $\mathrm{H}$, respectively [6]. The finder pattern is a pattern that detects the position of the QR code. You can perceive the information about the position, size, and angle of the symbol by placing this pattern at three corners of the symbol [7]. This finder pattern consists of structures that can be detected from every direction. The alignment pattern is a pattern to mend the distortion of the QR code. It is extremely effective in correcting nonlinear distortion. The center coordinates of the alignment pattern will be identified to mend the distortion of the symbol. For this purpose, an isolated black cell is placed in the alignment pattern so that the center coordinates of the alignment pattern can be easily detected. The timing pattern is a pattern to identify the center coordinates of each cell in a QR code in which black-and-white patterns are alternately arranged. It is used to correct the center coordinates of the data cell when the symbol is distorted or there is an error in the cell pitch. The arrangement of it is made in both the vertical and horizontal directions. The 2D bar code system is used in various fields. Wang proposed a scheme of embedding 2D barcodes into given Visual Cryptography shares to prevent cheating [8].

Radio frequency identification (RFID) is a technique for exchanging data between a reader and an electronic tag attached to a subject, using the radio wave communication for identification and tracking purposes. RFID has a high potential for the applications in areas such as supply chain management, access control via identification cards, and the asset tracking systems [9]. The RFID system is composed of a reader and tags, where an RF reader easily reads and writes data from each entity (RF tag). An RFID reader also supplies energy for tags using radio frequency, requests information on tags using tag ID, and interprets received signal from an ID-specified tag. RFID tags respond to readers and have unique identification information in the tag ID. RFID has been studied for various applications. Earlier studies showed RFID tag embedded in 2D barcode on PCB [10].

\section{Tagging Mechanisms}

Tagging mechanisms has been widely adopted for the administration of medicines in healthcare and medical applications. The embedded tags on medicine bottles or vials contain information that is essentially important not only for the medicine administration but also for the safety of the patients. It can contain both the manufacture and expiry dates of such medicines. Thus, embedded tags could guarantee the safety of the patients 
as well as the efficiency of medicine management.

Figure 2 shows the structure of tagging mechanism using barcodes. The barcode tag is made up of black and white lines which are embedded on medicine bottles. It can be immediately identified using a product database through a barcode reader. Reading of barcodes includes the following steps: (1) Barcode reader's scanning head shines its laser light or LED towards the barcode; (2) The light-detecting photoelectric cell of the barcode reader absorbs the reflected lights; (3) The reflected lights generates a code pattern of on-off pulses (e.g., black for 'off', white for 'on'); (4) These pattern of on-off pulses are transformed into binary digits (or decimal numbers); and (5) The generated binary digits (or decimal numbers) code are transmitted to the attached computer system for analysis and interpretation.

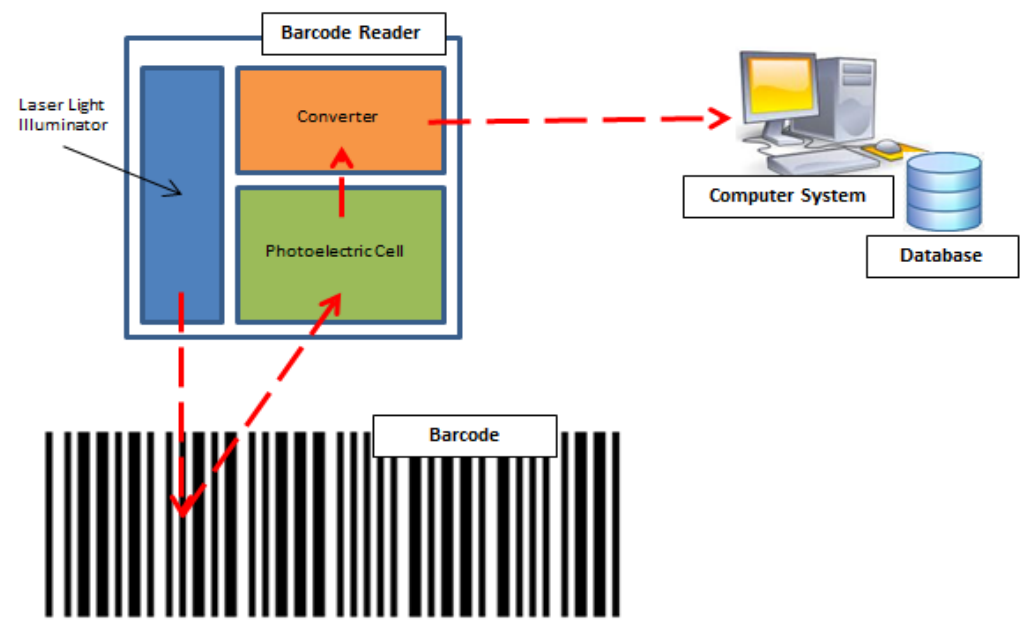

Figure 2. Barcode Tagging Mechanism

The RFID tagging mechanism has become a fast emerging enabling technology for healthcare systems and medicine administration applications. It refers to a non-contact wireless technology that utilizes electromagnetic fields in order to identify and track embedded tags from objects (e.g., medicine bottles, vials, etc.). The embedded information on tags is read by RFID readers through the transmission of radio frequencies. Its implementation on healthcare systems and medicine administration applications enhances the efficiency and effectiveness of gathering physiological information among patients and its relevant environment as well as for the administration of correct medicines for patients. The structure of RFID tagging mechanism is shown in Figure 3.

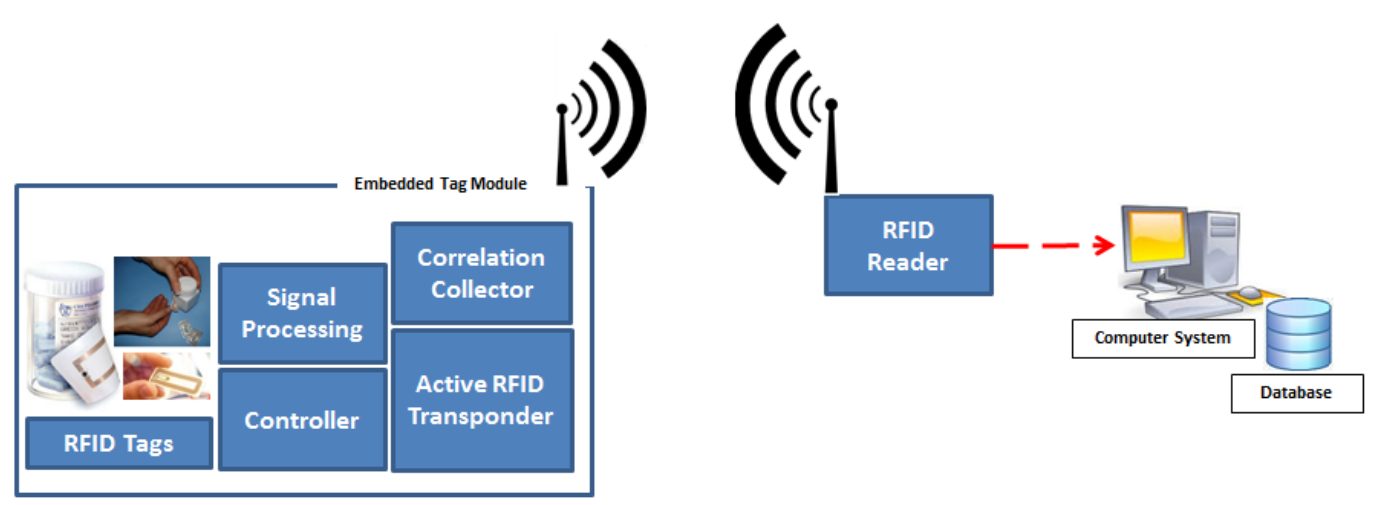

Figure 3. RFID Tagging Mechanism 
The RFID tagging mechanism provides an increased efficiency on monitoring and administering appropriate medicines for patients in healthcare systems and medical applications. It is mainly comprised of an embedded tag module, and RFID reader and antennas. These components work hand-in-hand with the interaction of the middleware that processes the tag signals and form it into single identification information. The embedded tags periodically transmits signals to the RFID reader (i.e., active tags) informing the current status of the medical object. Thus, current information from medicinal vials or bottles is frequently updated.

\section{Design}

In medical applications, administration of medicines is an important issue for patient safety. Medical applications have a strong need for embedded tags in vials to facilitate medicine management. Tags should be easy to recognize, even if the tag is attached to a curved surface for cylindrical or round type prescription bottles. Figure 4 shows how the original code suffers distortion when it is attached on cylinder type objects. The width of the code area becomes up to $2 / \pi$ times smaller as cylindrical length emerges.

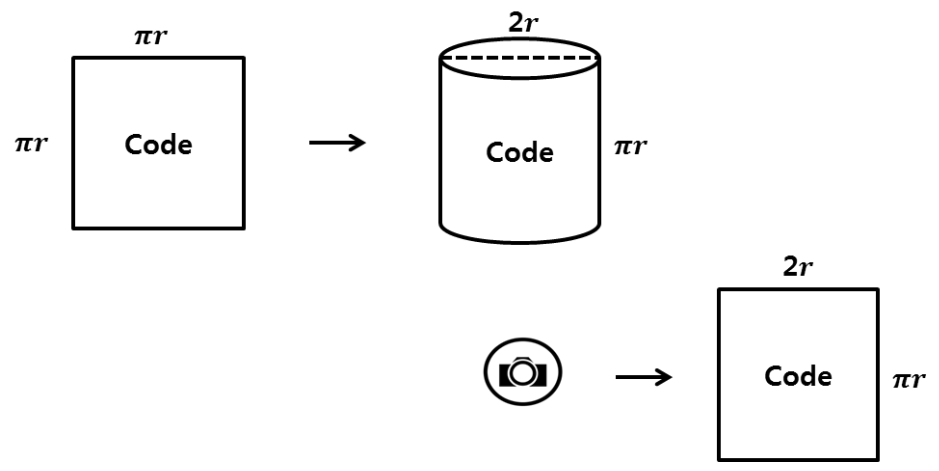

Figure 4. Distortion on Cylinder Type Object with Camera

Topology is a major branch of mathematics related to properties that are preserved even under the continual deformation of an object, such as an elongated but torn or bonded deformation. It emerged through the conceptual development from the disciplines of set theory and geometry, such as dimension, space and transformation. The set X, together with a collection $\mathrm{T}$ of its subsets, is called a topology when the subsets of $\mathrm{T}$ comply with the following properties: 1 . There are the empty set and the (trivial) subsets $\mathrm{X}$ in $\mathrm{T} ; 2$. Every time sets $\mathrm{A}$ and $\mathrm{B}$ are in $\mathrm{T}$, their intersection is in $\mathrm{T}$ as well. Every time two or more sets are in $\mathrm{T}$, their union is in $\mathrm{T}$ as well. In topology and related areas of mathematics, the neighborhood is one of the basic concepts in the field of topology space. Intuitively, a neighborhood of a point is a set including the point where you can move that point for a certain distance without leaving the set [12]. Two continuous map $f, g$ : $\mathrm{X} \rightarrow \mathrm{Y}$ are called homotopic ( $\mathrm{f} \sim \mathrm{g}$ ). In case that $\mathrm{f}$ can be unceasingly deformed into $\mathrm{g}$ in the class of continuous maps, if there is a one-parameter family of continuous map beginning with $\mathrm{f}$ and ending with $\mathrm{g}$ [14]. If $\mathrm{k}: \mathrm{X} \rightarrow \mathrm{Y}$ is an unceasing map, and in case that $\mathrm{F}$ is a path homotopy in $\mathrm{X}$ between the paths $\mathrm{f}$ and $\mathrm{f}$, then $\mathrm{k} \circ \mathrm{F}$ is a path homotopy in $\mathrm{Y}$ between the paths $\mathrm{k} \circ \mathrm{f}$ and $\mathrm{k} \circ \mathrm{f}$ in Figure 5 [15]. 


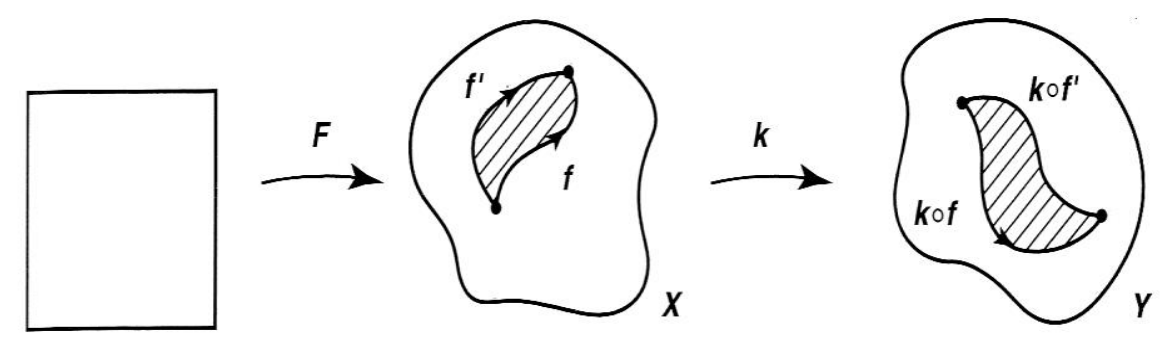

Figure 5. Homotopy

A topology-based approach is utilized in this paper, and graph matching for tag identification on curved surface were used. A CDPC which utilizes topological matching was used. Figure 6 shows the overall structure of CDPC. CDPC recognition scheme includes the following 5 components: (1) image capture with camera, (2) dot-pattern detection using Harris corner detector, (3) template search, (4) projective distortion correction and (5) data extraction [11]. First, the code image is captured using a low cost and low quality camera. Second, the system detects the code information dot using filtering and graph mapping. Third, the system matches the template using a Depth First Search. Fourth, an image transformation correction is performed using topology-based techniques. Lastly, data are extracted using this information and decoding process-based Reed-Solomon code [13].

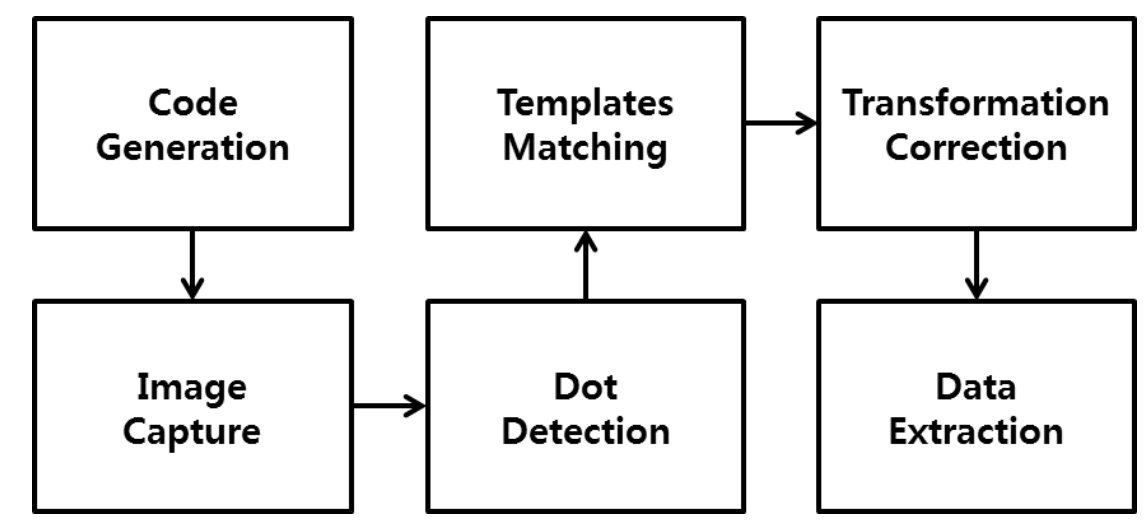

Figure 6. Overall Structure of CDPC

Figure 7 shows a diagram of three-shell type of CDPC (the number of shells can be extended when more data capacity is required). The centerline was set as a template for projective transformation correction. There are centerpoint and sine, and cosine cost is zero of each shell. Templates help in detecting and recognizing image rotation, and in determining the skew from the camera projection. The centerline template and side two points on the three shells were used to determine the lining. These three-shell data type CDPC can be inserted 42 binary bits. 


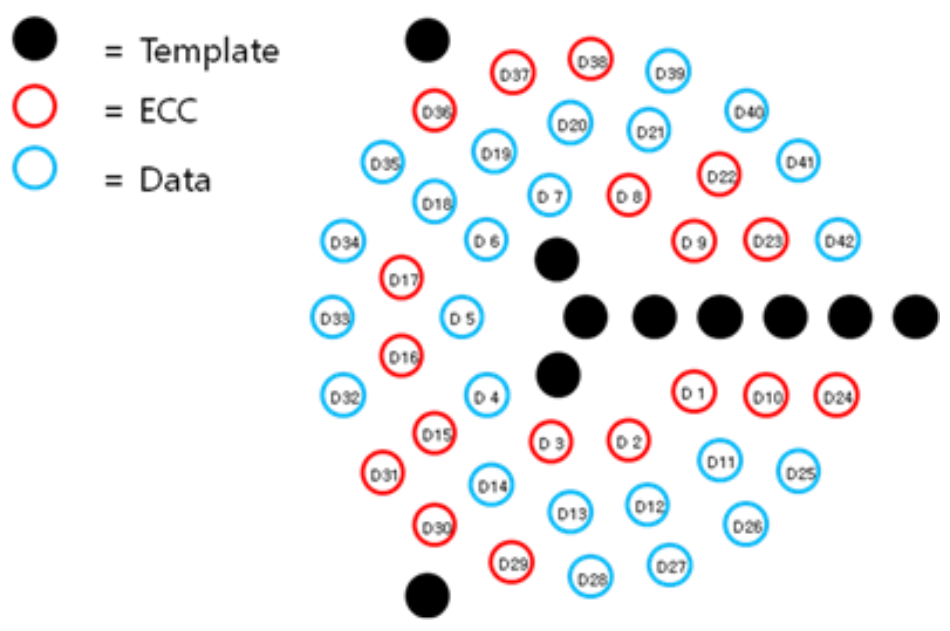

Figure 7. Structure of Three-shell CDPC

Several problems should be considered when detecting pattern codes. Geometric distortion can occur depending on the scanning code location. In order to recover geometric distortion, CDPC (1) uses baseline shell to find for reference dots, (2) starts to create a graph for the dots on curved surface, (3) and searches graph templates to correct location and information. Figure 8 shows the topology conservation under geometric distortion or projection on curved surface. The relationships between the dots still remained (invariant), and it is the key idea of CDPC to recover information from the invariants under any kind of geometric transformations. Basically, the inverse function of homotopy was computed in order to find the invariant.

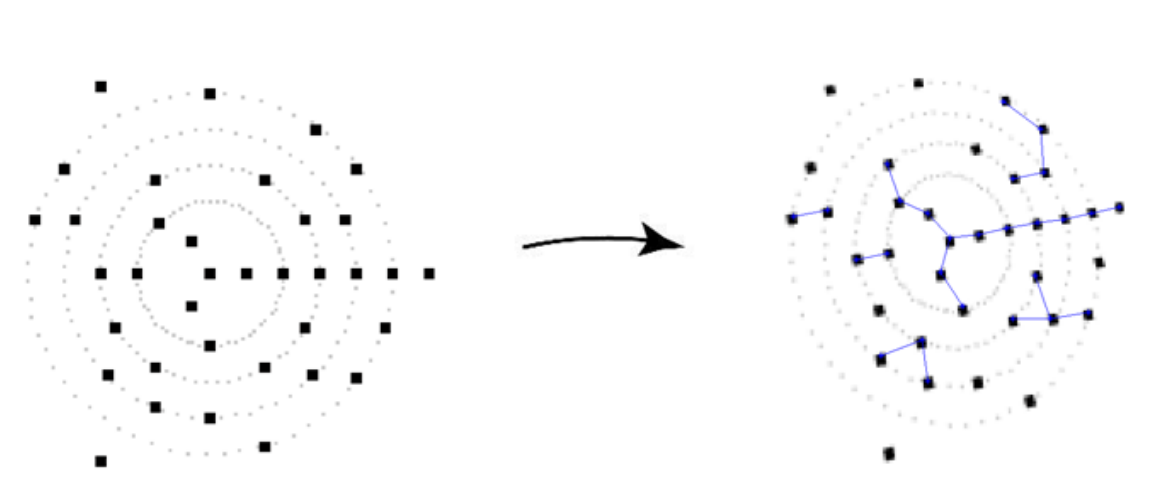

Figure 8. CDPC Transformation and Maintaining of Templates Relationships

\section{Experimental Results}

In this paper, the major feature of the system is cylindrical and round type prescription bottles tag. The system performance was tested for code pattern recognition on cylinder type objects. The cylinder object is tagged with QR codes and CDPC, and it is tested in terms of the performance of its recognition. Figure 9(a) shows QR code tagging on a cylinder object. QR code cannot work well on curved surface, because QR code is hard to recognize under cylinder type distortion. Figure 9(b) shows CDPC tagging with cylinder object, and CDPC can work well because CDPC recognition is basically topological dot matching, which is robust under cylinder type of distortion. 

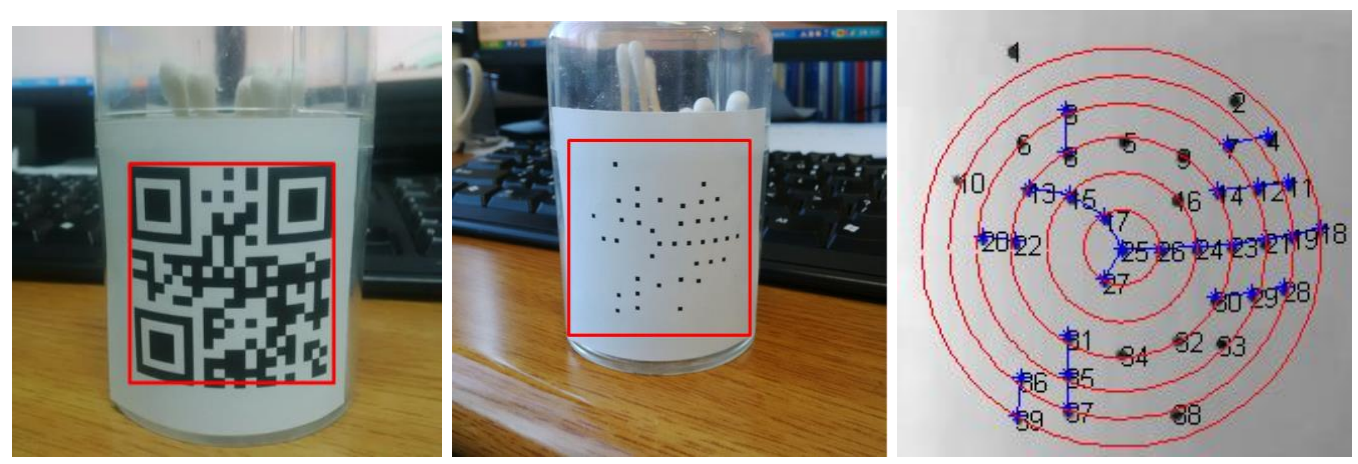

Figure 9. (a) Recognition Test of QR Code on Cylinder type object, and (b) Recognition Test of CDPC on Cylinder Type Object

Figure 10 shows QR code and CDPC recognition result on cylinder type object. The code width takes 1:1 1: $2 / \pi$ reduction rate with 0.01 intervals. Experiment was repeated 100 times for each interval. CDPC has good recognition results before 0.8 , while CDPC neighborhood relationship is destroyed after 0.8 . $\mathrm{X}$-axis is width reduction rate and Yaxis is code recognition rate.

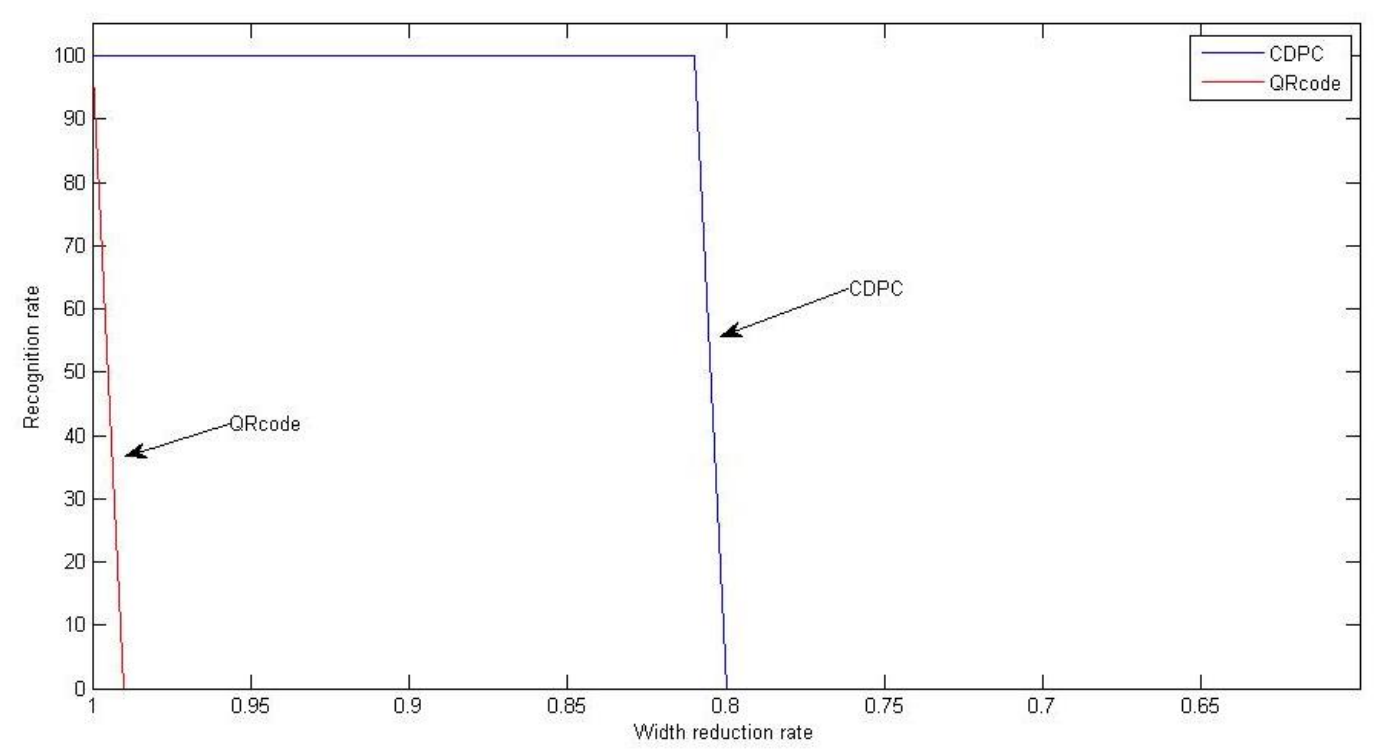

Figure 10. Experimental Results of QR and CDPC on Cylinder Object

\section{Conclusions}

Medical applications have a need for embedded tags on medical bottles to facilitate medicine management and user safety. The use of embedded tags enhances the efficiency of medicine management and guarantees the safety on administering appropriate medicines for patients. A 2D barcode such as square-based QR code is hard to recognize on cylindrical and round type prescription bottles, while RFID can easily break or damage on cylindrical and round type bottles. Experiments were conducted for tag recognition on cylindrical type bottles with QR code and CDPC. Topology-based CDPC recognition system has shown a good performance on cylindrical and round types of prescription bottles. Furthermore, experimental results show that CDPC outperformed 2D barcodes in recognition rate on cylinder objects. In the future, a CDPC for embedding 
information on 3D printing products for 3D object identification and management will be developed.

\section{Acknowledgments}

This work was supported by the 2014 Research Fund of the University of Seoul.

\section{References}

[1] U. K. Jain and S. Nayak, "Hyderabad: Pharma Med Press 1st ed", Pharmaceutical Packaging Technology, (2008), pp. 1-273.

[2] 2015-74, Notice of Ministry of Health and Welfare, "Use and management of drug barcodes and RFID tags, Partial amendments", (2015).

[3] T. Pavlidis, J. Swartz and Y. P. Wang, "Fundamentals of Bar Code Information Theory", IEEE Computer, IEEE Computer Society, vol. 23, no. 4, (1990), pp. 74-86.

[4] International Organization for Standardization. Information Technology - Automatic Identification and Data Capture Techniques - Bar Code Symbology - QR Code, ISO/IEC 18004, (2000).

[5] Denso Wave Inc. (2014) QRcode.com - Many ways of using QR Codes. http://www.qrcode.com/en/about/howtouse.html, (accessed 15 Feb 2015).

[6] QRStuff.com - QR Code Error Correction, http://blog.qrstuff.com/2011/12/14/qr-code-error-correction, (accessed 10, Jan. 2017).

[7] L. Yue and L. Mingjun, "Automatic recognition algorithm of quick response code based on embedded system, Proceedings -ISDA: Sixth International Conference of Intelligent Systems Design and Applications, (2006).

[8] G. Wang, F. Liu and W. D. Yan, "2D Barcodes for visual cryptography", Multimedia tools and applications, vol. 75, no. 2, (2016), pp. 1223-1241.

[9] K. Finkenzeller, "RFID Handbook: Fundamentals and Application in Contact-less Smart Card and Identification 2nd edition", John Wiley \& Sons Ltd. (2003).

[10] J. Han, G. Wang and J. Sidén, "Fragment-type UHF RFID tag embedded in QR barcode label", Electronics Letters, vol. 51, no. 4, (2015), pp. 313-315.

[11] J.-Y. Shim and S.-W. Kim, "Design of circular dot pattern code (CDPC) for maximum information capacity and robustness on geometric distortion/noise", Multimedia Tools and Applications, vol. 70, no. 3, (2014), pp. 1941-1955.

[12] S. Lynn Arthur and S. Arthur J., "Counterexamples in Topology", Springer, Berlin, (1978).

[13] T. K. Moon, "Error Correction Coding: Mathematical Methods and Algorithms", John Wiley \& Sons, (2005), pp. 293-368.

[14] V. A. Vassiliev, "Introduction to Topology", American Mathematical Society, (2001).

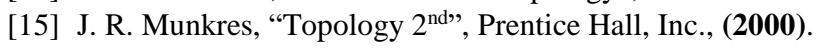


International Journal of Control and Automation Vol.10, No.4 (2017) 